\title{
EVALUACIÓN DEL IMPACTO EN LA CALIDAD DE AGUA DEBIDO A LA PRODUCCIÓN SEMI INTENSIVA DE TRUCHA (Oncorhynchus mykiss) EN JAULAS FLOTANTES EN LA LAGUNA ARAPA - PUNO
}

Wilfredo Vásquez Quispesivana*a, María Talavera Núñez ${ }^{\text {, }}$ Marianela Inga Guevara

\section{RESUMEN}

La investigación se realizó en la laguna Arapa, ubicada en Puno, se determinaron parámetros físico químicos y un modelo matemático simplificado para el fósforo. Se utilizó el diseño experimental "BACI" (Before-After-Control-Impact) para evaluar el impacto en la calidad del agua. Durante la etapa "Después-Impacto", se realizaron cinco siembras de 70000 truchas cada tres meses, registrándose consumo de alimento e incremento de biomasa, en este periodo se manifestaron diferencias significativas en acidez, dióxido de carbono, fosfatos y conductividad eléctrica. Los sólidos suspendidos totales demostraron una disminución. La alcalinidad, pH y oxígeno disuelto permanecieron constantes. Se calculó que $611 \mathrm{~kg}$ de fósforo fueron vertidos a la laguna, producto de la digestión del alimento, posteriormente se estableció el modelo matemático simplificado para determinar la evolución del fósforo disuelto en la columna de agua en función del tiempo; se determinó que de $611 \mathrm{~kg}$ vertidos a la laguna, $246 \mathrm{~kg}$ se destinó a acumularse en los sedimentos y $365 \mathrm{~kg}$ se fueron disolviendo en todo el volumen de agua. La concentración de fósforo en la laguna Arapa se incrementó con la actividad de crianza de truchas, alcanzando valores de $32,79 \mathrm{mg} / \mathrm{m}^{3}$ de PO4-P que lo clasifican como lago eutrófico según la clasificación de Vollenweider

Palabras clave: Truchas, laguna, fósforo, modelo matemático, diseño BACI.

\section{EVALUATION OF IMPACT ON WATER QUALITY DUE TO THE SEMI INTENSIVE PRODUCTION OF TROUT (Oncorhynchus mykiss) IN FLOATING CAGES IN THE ARAPA LAKE - PUNO}

\begin{abstract}
This research was developed in the Arapa lake, located in Puno, physicochemical parameters and a simplified mathematical model for the phosphorus were evaluated. Experimental design "BACI" (Before-After-Control-Impact) was used to assess the impact on water quality. During the "After-Impact" period, 70000 trouts were farmed every 3 months; then the food

\footnotetext{
a. Facultad de Pesquería, Universidad Nacional Agraria La Molina, Av. La Molina S/N, Lima 12, Perú, wvasquez@lamolina.edu.pe

b. Facultad de Ciencias Naturales y Formales, Universidad Nacional de San Agustín de Arequipa.

c. Facultad de Industria Alimentarias, Universidad Nacional Agraria La Molina
} 
consumed and increased biomass was registered for each batch of trout farmed. In this period significant difference in acidity, carbon dioxide, phosphates and electrical conductivity was expressed. Total suspended solids showed a decrease. Alkalinity, $\mathrm{pH}$ and dissolved oxygen remained constant. Product of the digestion of food $611 \mathrm{~kg}$ of phosphorus were discharged into the Arapa lake; then the simplified mathematical model was established to determine the evolution of dissolved phosphorus versus time. From de $611 \mathrm{~kg}$ of phosphorus discharged into the Arapa lake, $246 \mathrm{~kg}$ were directed to accumulate in sediment and $365 \mathrm{~kg}$ were dissolving in the entire volume of water. The concentration of phosphorus in the Arapa lake increased with trout farming activity, reaching values of $32.79 \mathrm{mg} / \mathrm{m}^{3}$ of PO4-P to classify it as eutrophic lake as rated by Vollenweider.

Key words: Trout, lake, phosphorus, mathematical model, BACI design

\section{INTRODUCCIÓN}

Las nuevas tendencias que describen a la acuicultura como una actividad económica en aumento convergen en definirla como el manejo de la calidad del agua para la cría de organismos acuáticos en cautividad con fines comerciales. Esta actividad es susceptible a la degradación del medio ambiente debido a la utilización del agua que recibe grandes cantidades de desechos, como el alimento no consumido por los peces y las heces. Se introducen sustancias químicas al ecosistema; además, una significativa porción de los nutrientes queda disuelta en la columna de agua, produciendo fenómenos de eutrofización. Este impacto tiene un costo ambiental, económico y social ${ }^{1}$.

El alimento balanceado es el principal insumo utilizado en el cultivo intensivo de peces; la calidad de sus ingredientes, así como el tipo de procesamiento, determinará la eficiencia del alimento; sin embargo, las prácticas adoptadas de alimentación que determinan el cálculo y cantidad a dar a los peces, repercutirá significativamente en los aspectos de productividad, económicos y medioambientales.

El fósforo es considerado como uno de los elementos más limitantes en la alimentación de los animales acuáticos; puede ser absorbido directamente del medio acuático, pero las concentraciones, generalmente, son muy limitadas; la asimilación de fósforo a partir del alimento es 200 veces mayor que la del absorbido del agua. Los requerimientos mínimos para un normal crecimiento en trucha arco iris es de 7 - 8 gramos de fósforo $/ \mathrm{kg}$ de alimento ${ }^{2}$.

La asimilación del fósforo a partir de los minerales agregados a la dieta es bastante elevada y suele ser de alrededor de 90 a $95 \%{ }^{3}$.

Según Restrepo ${ }^{4}$ los primeros modelos matemáticos que se desarrollaron consistió en expresiones analíticas simples, que se utilizaron para calcular la concentración de cierta sustancia en función del tiempo, en sistemas naturales muy simplificados como lagos completamente mezclados y ríos unidimensionales. El trabajo de Streeter y Phelps5 sobre 
las variaciones de oxígeno disuelto, a lo largo de un río, en función de la descarga de materia orgánica, es pionero y un clásico en este tiempo. En la actualidad, los modelos de calidad de aguas están enfocados principalmente hacia el estudio de la eutrofización, problemas derivados del aumento de la producción primaria causada por la abundancia de nutrientes $u$ otros factores.

La mayoría de los modelos descritos en la literatura tratan con la dinámica de una sustancia en un cuerpo de agua bien mezclado verticalmente. Por otro lado, muchos constituyentes toman lugar en procesos biológicos y químicos que están sujetos a las transformaciones debido a estratificación térmica de la columna de agua ${ }^{4}$.

El acercamiento básico de varios de los modelos es el balance de masa del nutriente limitante asumido. Se usa la variable fósforo total como la variable indicadora del estado trófico.

\section{PARTE EXPERIMENTAL}

\section{Zona de experimentación}

El presente trabajo se realizó en la zona de producción de trucha en jaulas flotantes en la laguna Arapa, ubicado en la comunidad de Iscayapi, distrito de Arapa, provincia de Azángaro, departamento de Puno. Las coordenadas del área de trabajo se indican en la tabla 1.

Tabla 1. Coordenadas del área de trabajo

\begin{tabular}{ccc}
\hline Puntos & Latitud - Sur & Longitud - Oeste \\
\hline A & $15^{\circ} 8^{\prime} 36.46^{\prime \prime} \mathrm{S}$ & $70^{\circ} 4^{\prime} 39.09^{\prime \prime O}$ \\
B & $15^{\circ} 8^{\prime} 15.57^{\prime \prime S}$ & $70^{\circ} 4^{\prime} 38.77^{\prime \prime} \mathrm{O}$ \\
\hline
\end{tabular}

\section{Diseño experimental BACI (Before-After - Control-Impact)}

Este diseño comprende muestreos y evaluaciones antes y después del impacto, tanto en el área impactada como en la zona control (figuras la y 1b). Las jaulas flotantes se instalaron en la zona B, mientras que la zona A permaneció como zona control, donde no se ha instalado jaulas flotantes en todo el periodo de experimentación. Se realizó 8 evaluaciones antes y después del impacto en cada sitio (zonas A y B).

\section{Evaluación de la calidad de agua}

Los análisis físicos y químicos del agua de la laguna Arapa se realizaron in situ utilizando un kit de análisis de agua HANNA instruments ${ }^{\circledR}$. 


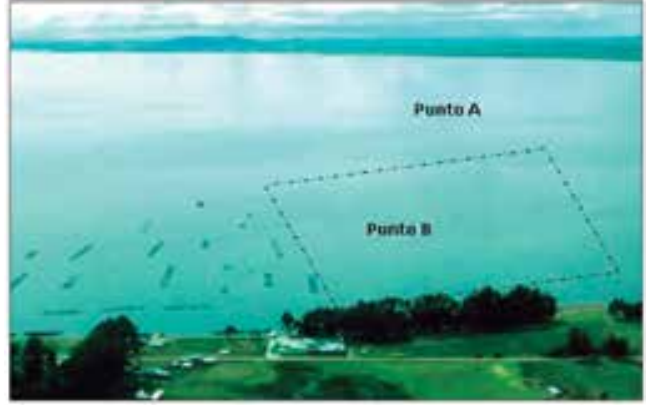

(a)

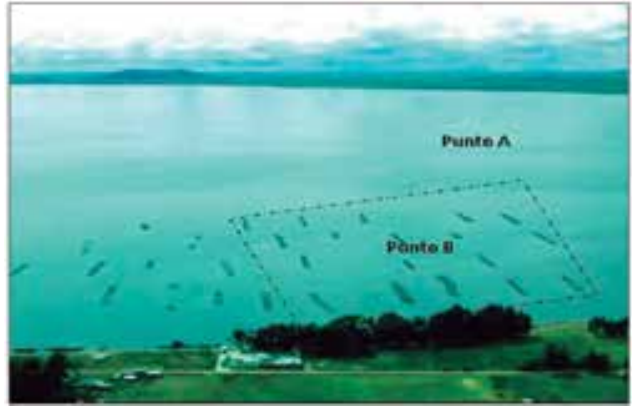

(b)

Figura 1. Puntos de muestreo Diseño BACI: (a) Durante 14 meses se evaluó 8 muestras en ambas zonas A y B antes del impacto, (b) después de instalar las jaulas flotantes (impacto) en la zona B y mantener a la zona A como control; durante otros 14 meses se evaluó 8 muestras más en ambas zonas A y B.

Se utilizó el software IBM® SPSS Statistics para el análisis de los datos obtenidos. Se aplicó un análisis de varianza de dos factores.

Modelo matemático simplificado del fósforo, según Thomann y Mueller ${ }^{6}$ se puede hacer un modelo del comportamiento del fósforo en un lago ideal, en el que se asume una serie de simplificaciones para aplicar un equilibrio de masas (figura 2).

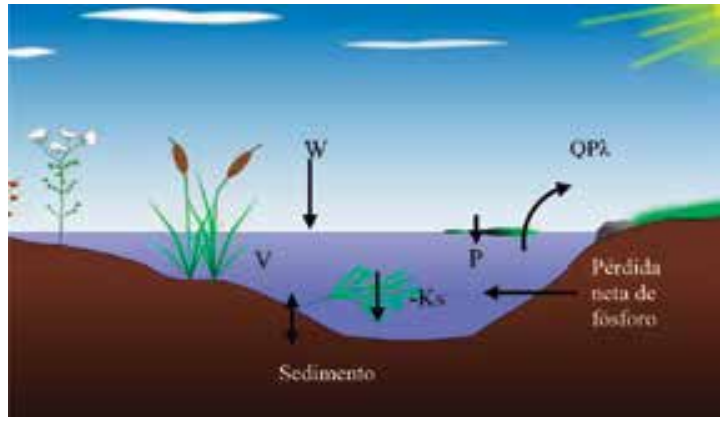

Donde:

$\mathrm{V}=$ Volumen del lago $\left[\mathrm{L}^{3}\right]$

$\mathrm{P} \lambda=$ Fósforo (total) en el lago, $\left[\mathrm{M} / \mathrm{L}^{3}\right]$

$\mathrm{W}=$ Fuentes externas de fósforo, $[\mathrm{M} / \mathrm{T}]$

$\mathrm{Ks}=$ Tasa de pérdida global de fósforo total $[\mathrm{I} / \mathrm{T}]$

Figura 2. Esquema del balance de masa para el fósforo, lago completamente mezclado

El análisis para el balance de contaminantes sobre una escala de tiempo anual considera al cuerpo lentico como un reactor de mezcla completa (análisis cero dimensional). El modelo simplificado tiene en cuenta el balance entre el ingreso y la disposición del fósforo en relación a la tasa de sedimentación:

\section{Donde:}

$$
\frac{\mathrm{dC}}{\mathrm{dt}}=\mathrm{Qe} * \mathrm{Ce}-\frac{\mathrm{kC}}{\mathrm{V}}
$$


$C$ : concentración media del contaminante en la laguna en $\mathrm{kg} / \mathrm{m}^{3}$

Qe: caudal ingresante en $\mathrm{m}^{3} /$ día $^{2}$

$\mathrm{Ce}$ : concentración de fósforo en el flujo ingresante en $\mathrm{kg} / \mathrm{m}^{3}$

$K=$ tasa de sedimentación o tasa de remoción $0,36 /$ día $^{7}$

\section{RESULTADOS Y DISCUSIÓN}

\section{Evaluación de la calidad de agua}

En la figura 3 se observa la evolución temporal de los parámetros de calidad de agua medidos durante los dos periodos de estudio; Antes y Después del Impacto (instalación y crianza de trucha en jaulas flotantes) en las zonas Control e Impactada.

Durante la etapa "Después-Impacto" correspondiente al diseño BACI, se manifestaron diferencias significativas en los parámetros de acidez (3a), dióxido de carbono (3c), y conductividad eléctrica ( $3 \mathrm{~g})$; incrementándose los valores de estos parámetros. La acidez, se incrementó en la zona de impacto en el periodo posterior a la instalación de jaulas flotantes hasta valores de $9 \mathrm{mg} / \mathrm{L} \mathrm{CaCO}_{3}$; esto coincide con la elevada carga de truchas sembradas y valores de $\mathrm{pH} 8,7$ registrados, lo que sugiere la existencia de una elevada descomposición de materia orgánica carbónica ${ }^{8}$. Mientras que la acidez en la zona control permaneció constante entre 7,7 y $8,0 \mathrm{mg} / \mathrm{L} \mathrm{CaCO}$. $\mathrm{López}^{8}$ reportó valores de acidez entre 5 y $10 \mathrm{mg} / \mathrm{L} \mathrm{CaCO}$ indicando que es uno de los parámetros químicos que no se alteran por la crianza de trucha.

En relación al $\mathrm{CO}_{2}$, se observa que los valores obtenidos en la zona impactada son siempre mayores (rango de 5 a $6,7 \mathrm{mg} / \mathrm{L}$ ) que la zona control; la diferencia es más notoria en el periodo posterior a la instalación de jaulas y crianza de truchas. Según Blanco ${ }^{9}, \mathrm{el} \mathrm{CO}_{2}$ presente en el agua procede principalmente de la respiración de los animales y plantas que viven en ella, así

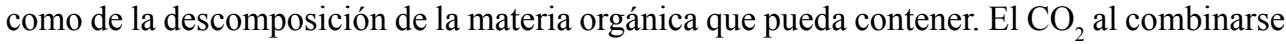
con el agua forma ácido carbónico $\mathrm{H}_{2} \mathrm{CO}_{3}$ (ácido débil, muy inestable que tiende a disociarse en $\mathrm{CO}_{2} \mathrm{y} \mathrm{H}_{2} \mathrm{O}$ ) que acidifica el medio, pero dependiendo del contenido en bases de calcio y magnesio del agua, forma carbonatos y bicarbonatos.

Con respecto a la conductividad, se puede indicar que la temperatura es una variable que modifica sensiblemente los valores de este parámetro ${ }^{10}$. Con valores que van desde 543 a $853 \mu \mathrm{S} / \mathrm{cm}$ se consideran a las aguas de la zona de estudio como apto para la crianza de trucha. Sin embargo, Blanco ${ }^{9}$ señala que la mayoría de las especies acuáticas soportan bien las distintas concentraciones de sales disueltas en el agua. La conductividad de las aguas de lago generalmente es baja; puede variar entre 50 y $1500 \mu \mathrm{S} / \mathrm{cm}$; valores que excedan estos rangos pueden indicar que el agua no es adecuada para la vida de ciertas especies de peces. Por lo tanto la conductividad es una medida generalmente útil como indicador de la calidad de aguas dulces.

La evolución temporal de los sólidos totales disueltos (STD) que se representa en la figura 3(h), demuestra una disminución de los valores (de 435 a $270 \mathrm{mg} / \mathrm{L}$ ) que son notorios en el 
segundo periodo del estudio (después de instalar las jaulas flotantes e iniciar la crianza de trucha), estos valores se encuentran por debajo del límite de $500 \mathrm{mg} / \mathrm{L}$ que recomienda los estándares de calidad ambiental para agua de categoría 4. Desde el punto de vista limnológico, las concentraciones de STD guardan una correlación positiva con la productividad en lagos. Al mismo tiempo los STD afectan la penetración de luz en la columna de agua y la absorción selectiva de las diferentes longitudes de onda que integran el espectro visible. En el caso de los cuerpos de agua donde se realiza la crianza de truchas, el alimento no ingerido, las partículas finas de alimento, las heces de las truchas, las algas o películas bacterianas desprendidas del sistema jaulas, se acumulan en el medio acuático inmediato a los sistemas de cultivo, constituyendo los desechos sólidos. Su concentración puede influir sobre todos los demás procesos del sistema, constituyendo una fuente importante de empleo o demanda de oxígeno e incorporación de nutrientes al agua y pueden afectar la salud de los peces actuando sobre su sistema branquial y aumentando su exposición a los patógenos.

Referente a los parámetros de alcalinidad, $\mathrm{pH}$, oxígeno disuelto y fosfatos, éstos permanecieron constantes. Los valores de alcalinidad reportados y que se representan en la figura ( $3 b)$, permanecen constantes en un rango de 80 a $90 \mathrm{mg} / \mathrm{L} \mathrm{CaCO}_{3}$. Según Cole ${ }^{11}$, las fluctuaciones diarias de la alcalinidad son despreciables. En aguas diluidas los efectos de la fotosíntesis y de la respiración en el sistema del ácido carbónico están marcados principalmente por los cambios de $\mathrm{pH}$. El incremento del $\mathrm{CO}_{2}$ baja el $\mathrm{pH}$ sin cambiar mucho la alcalinidad. En los casos que se produce una elevación y disminución estacional de los valores de alcalinidad en lagos de aguas duras carbonatadas, se observa una disminución de la alcalinidad del epilimnio (capa cálida superior de agua en un lago) durante la temporada de crecimiento debido a que la incorporación de $\mathrm{CO}_{2}$ provoca la precipitación de $\mathrm{CaCO}_{3}$. El carbonato que se hunde es redisuelto en el hipolimnio (capa inferior de agua en un lago) por el ácido carbónico formado por la hidratación del $\mathrm{CO}_{2}$ de la degradación y retorna al epilimnio durante la remoción siguiente.

Lópe ${ }^{8}$ reportó que el pH es uno de los parámetros que no se alteran por la crianza de trucha. Esta afirmación se valida con los resultados obtenidos; en la figura (3d) se observa que no existen diferencias estadísticamente significativas entre los factores de ubicación y tiempo. Según Klontz ${ }^{12}$ los valores de $\mathrm{pH}$ que se consideran aceptables para la crianza de truchas están entre 6,7 y 8,5. Uno de los productos finales del metabolismo proteico de las truchas, es el amoniaco. El amoniaco es un producto tóxico, con carácter limitante en su forma no ionizada $\left(\mathrm{NH}_{3}\right)$, la cual, bajo la acción de $\mathrm{pH}$ ácido se transforma en ion amonio $\left(\mathrm{NH}_{4}^{+}\right)$forma ionizada, no tóxica, lo que no ocurre en condiciones básicas ${ }^{9}$.

En relación al oxígeno disuelto (con un valor mínimo de $5 \mathrm{mg} / \mathrm{L}$ y un máximo de $6 \mathrm{mg} / \mathrm{L}$ ) presenta una evolución temporal homogénea como se representa en la figura (3e) donde se aprecia una distribución similar en ambas zonas de estudio. Los valores registrados guardan relación con la altitud de la zona de estudio; se conoce que la presión atmosférica varía con la altitud, latitud y condiciones climáticas. Cuanto menor sea la altitud sobre el nivel del mar, mayor será la presión atmosférica y mayor será la capacidad que tiene el agua para disolver oxígeno. La altura produce una disminución significativa del contenido de oxígeno disuelto 
en el agua y se ha encontrado que a alturas superiores a $3500 \mathrm{msnm}$, representa el 65\% del valor que se puede encontrar en la costa (nivel del mar). Como referencia, generalmente para los cuerpos de agua de la región altiplánica, principalmente para el lago Titicaca, el rango normal de oxígeno disuelto oscila entre 4,5-7,5 mg/L. Los valores encontrados disminuyen en los meses de verano, cuando la temperatura del agua supera los $19,0^{\circ} \mathrm{C}$, donde pueden presentarse concentraciones por debajo de $5,0 \mathrm{mg} / \mathrm{L}$, generando estrés en la trucha por las dificultades para extraer el oxígeno del agua y transportarlo a través de sus branquias.

La temperatura del agua influye directamente sobre la biología de las truchas; principalmente sobre el ritmo mensual de crecimiento y el grado de actividad metabólica. De manera indirecta influye en el agua del lago, sobre la concentración de oxígeno disuelto, la concentración de productos metabólicos (amoníaco) y el tiempo y grado de descomposición de los materiales depositados en el fondo.

Concerniente a los fosfatos, en las aguas puras son muy poco abundantes, pero pueden ser muy manifiestos en aquellas que recogen vertidos urbanos por la presencia de polifosfatos, que componen los detergentes o bien, en las que arrastran abonos agrícolas no consumidos. Los fosfatos no son tóxicos para las truchas, pero dan origen a una gran proliferación vegetal ${ }^{9}$. En la figura (3f) los valores recolectados entre 0,1 a $1,5 \mathrm{mg} / \mathrm{L}(100$ a $1500 \mu \mathrm{g} / \mathrm{l})$ de fosfatos no presentan diferencias estadísticamente significativas.

Los compuestos del fósforo son nutrientes de las plantas y conducen al crecimiento de algas en aguas superficiales. Dependiendo de la concentración de fosfato en el agua, puede producirse la eutrofización. $1 \mathrm{~g}$ de fosfato-fósforo $\left(\mathrm{PO}_{4}-\mathrm{P}\right)$ provoca el crecimiento de hasta $100 \mathrm{~g}$ de algas. Cuando estas algas mueren, los procesos de descomposición dan como resultado una demanda de oxígeno de alrededor de $150 \mathrm{~g}$. Las concentraciones críticas para una eutrofización incipiente se encuentran entre $0,1-0,2 \mathrm{mg} / \mathrm{L} \mathrm{PO}_{4}-\mathrm{P}$ en el agua corriente y entre $0,005-0,01 \mathrm{mg} / \mathrm{L} \mathrm{PO}_{4}-\mathrm{P}$ en aguas tranquilas ${ }^{13}$.

\section{Modelo matemático simplificado del fósforo. Determinación de la masa de contaminante (fósforo) ingresante por unidad de volumen del lago y por unidad de tiempo}

Se calculó la cantidad de fósforo que ingresa al cuerpo de agua, mediante las "declaraciones de impacto ambiental de fósforo" correspondientes al alimento balanceado para truchas (se tomaron los datos declarados por Biomar ${ }^{14}$ ). Las "declaraciones de impacto ambiental de fósforo" corresponden a la cantidad de nitrógeno y fósforo vertidos por cada kilogramo de biomasa producida, producto del consumo de alimento balanceado por las truchas, en el periodo de investigación.

En la tabla 2, se detalla la estimación de la cantidad de fósforo $(\mathrm{P})$ vertido a las aguas de la laguna Arapa (P/ton), la estimación se realizó en base a las declaraciones de impacto ambiental de $\mathrm{P}$ consignado en las fichas técnicas de cada tipo de alimento balanceado; los alimentos denominados "inicio" destinados para alevinos de trucha utilizan mayor cantidad de harina de pescado en su elaboración, con el objetivo de alcanzar porcentajes elevados 
de proteína $(58 \%)$, sin embargo, también son los que mayor cantidad de fósforo generan, producto de la digestión del alimento, como se indica en la tabla 2. Para la siembra de 70000 truchas realizadas en marzo del 2013 se ha consumido en el mismo mes de marzo, $1167 \mathrm{~kg}$ de alimento tipo inicio; con un factor de conversión (FC) de 1,0 se obtuvo un incremento de biomasa (BP) de $1167 \mathrm{~kg}$. El factor que corresponde para el alimento tipo inicio es de 11,5 $\mathrm{kg}$ de $\mathrm{P}$ vertido por tonelada de biomasa de trucha producida. Con estos datos se calculó un valor de 13,4 kg de P vertido al medio ambiente en el primer mes de crianza debido sólo a la siembra de marzo-2013.

Para el mismo lote o siembra de truchas realizadas en marzo del 2013 se observó que en el mes de agosto se ha consumido $3500 \mathrm{~kg}$ de alimento tipo engorde, con un factor de conversión (FC) de 1,1 se obtuvo un incremento de biomasa (BP) de $3182 \mathrm{~kg}$. El factor que corresponde para el alimento tipo engorde es de $7 \mathrm{~kg}$ de $\mathrm{P}$ vertido por tonelada de biomasa de trucha producida. Con estos datos se calculó un valor de $22,3 \mathrm{~kg}$ de $\mathrm{P}$ aportado al medio ambiente en el mes de agosto solo para la siembra de marzo 2013.

Durante el periodo que se produce el impacto (cultivo de truchas) se realizó 5 siembras de truchas (marzo 2013; junio 2013; septiembre 2013; diciembre 2013 y marzo 2014); cada siembra de 70000 truchas se cultiva por ocho meses hasta alcanzar un peso comercial y cosecharlas para la venta. El total de alimento consumido por estas siembras en conjunto fue de $70478 \mathrm{~kg}$ distribuidos en los diferentes tipos de alimento (inicio, crecimiento, engorde y acabado).

La cantidad total de $\mathrm{P}$ vertido al lago, producto de la digestión del alimento consumido, fue de $611 \mathrm{~kg}$, distribuidos mensualmente en función de la cantidad de la biomasa (65 $450 \mathrm{~kg})$ producida de trucha durante el tiempo de cultivo.

El fósforo principalmente procede del alimento; se ha determinado que el de origen vegetal se asimila mucho menos que el de origen animal. Una disminución del aporte en la ración $(0,6 \%$ es suficiente) permite reducir la cantidad de fósforo residual, lo cual es importante desde el punto de vista de la eutrofización ${ }^{15}$.

Para reducir la cantidad de fósforo se puede elegir un alimento que, dando los mismos resultados zootécnicos, ocasione menos residuos (los alimentos extruidos disminuyen los residuos en aproximadamente una tercera parte). Es casi imposible tratar el fósforo disuelto en el agua, tanto por las bajas concentraciones como por los elevados caudales. La única estrategia posible es reducir la dosis (en concreto la de la parte poco asimilable) en el alimento, sin que por ello se vean afectados ni la salud ni el crecimiento de los peces. Los fabricantes trabajan para comercializar alimentos de buena biodisponibilidad en fósforo y reducen los aditivos alimentarios que contienen fósforo soluble. En cuanto al productor, debe evitar las pérdidas de alimento durante el suministro. Los alimentos actuales sólo liberan 4,5 $\mathrm{kg}$ de fósforo por tonelada de peces producida, frente a los $31 \mathrm{~kg}$ que liberaban en 1994. El aumento del número de ingestas diarias parece favorecer mejora de la asimilación de los alimentos y por tanto reducir los residuos ${ }^{15}$. 


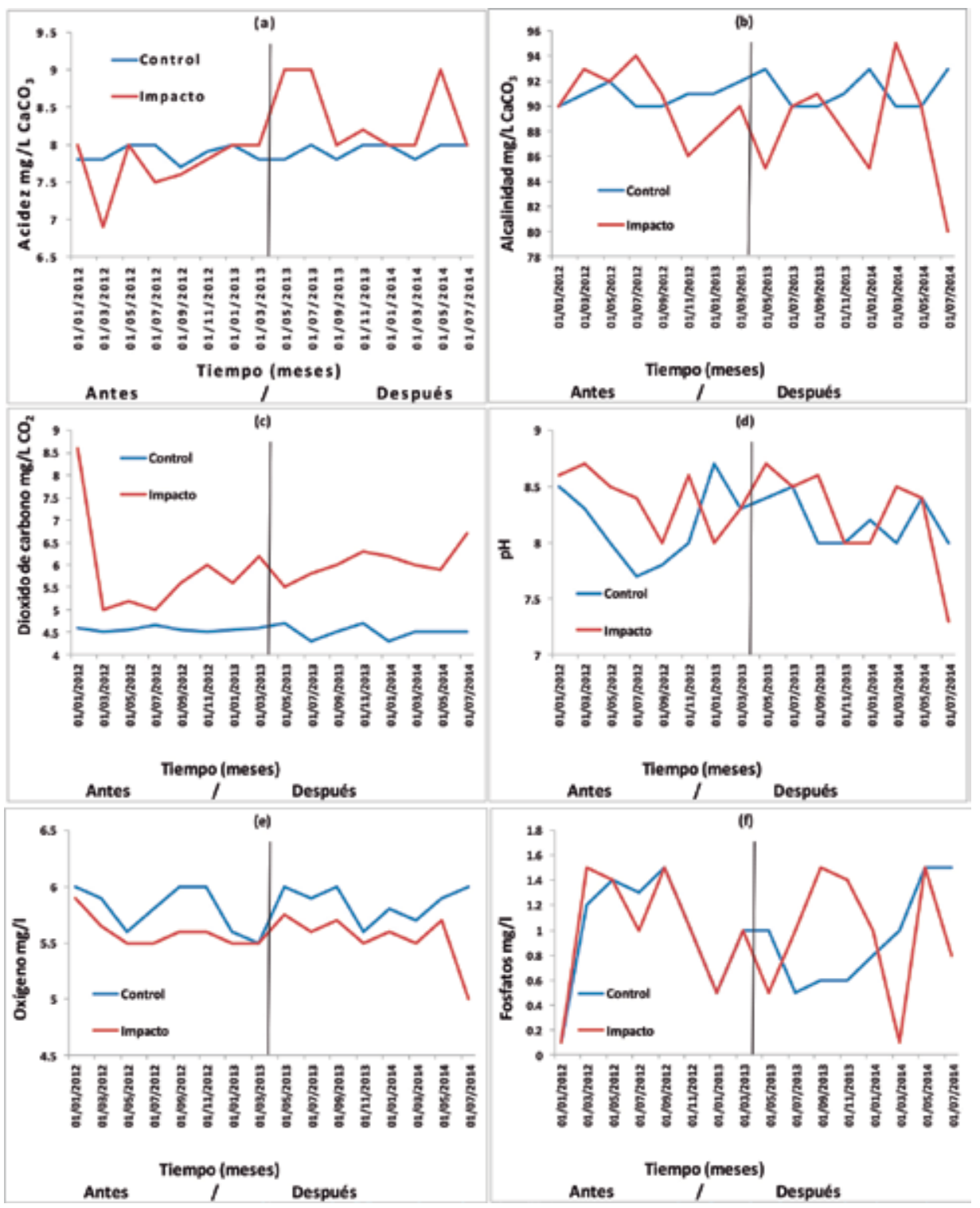

Figura 3. Evolución temporal de los parámetros de calidad de agua medidos durante los dos periodos de estudio; antes y después del impacto (instalación y crianza de trucha en jaulas flotantes) en las zonas control e impactada: (a) acidez; (b) alcalinidad; (c) dióxido de carbono; (d) $\mathrm{pH}$; (e) oxígeno y (f) fosfatos. 


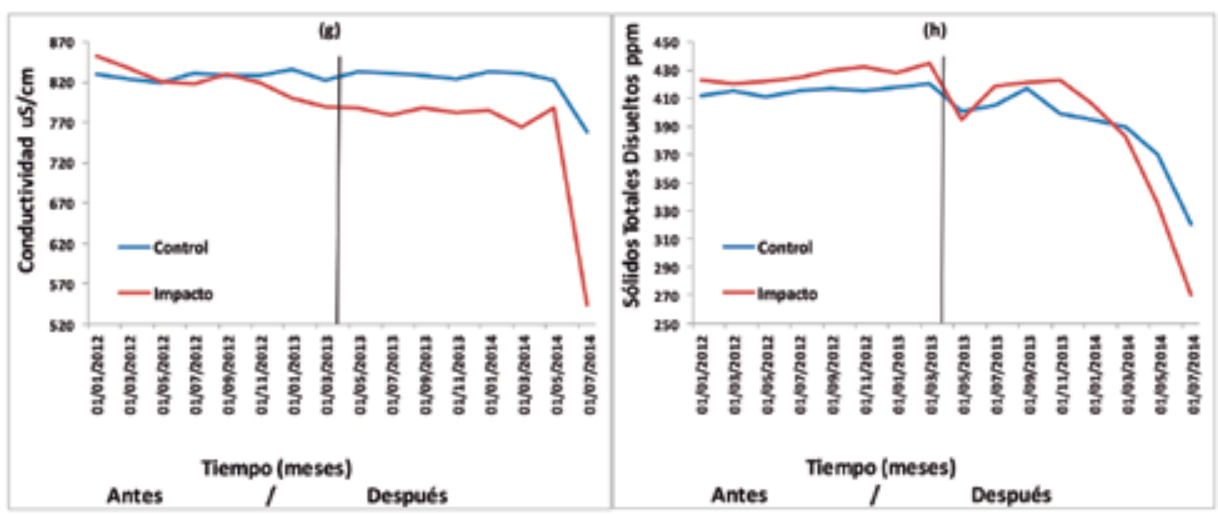

Figura 3 (Cont.). Evolución temporal de los parámetros de calidad de agua medidos durante los dos periodos de estudio; antes y después del impacto (instalación y crianza de trucha en jaulas flotantes) en las zonas control e impactada: (g) conductividad y (h) sólidos totales disueltos.

\section{Balance de fósforo en la laguna Arapa}

Sobre una escala de tiempo anual se consideró a la laguna Arapa como un reactor de mezcla completa. El modelo simplificado tiene en cuenta el balance entre el ingreso y la disposición del fósforo en relación a la tasa de sedimentación:

Donde:

$$
\frac{\mathrm{dC}}{\mathrm{dt}}=\mathrm{Qe} * \mathrm{Ce}-\frac{\mathrm{kC}}{\mathrm{V}}
$$

$c$ : concentración media de $\mathrm{P}$ en el lago en $\mathrm{kg} / \mathrm{m}^{3}=3,268 \times 10-5 \mathrm{~kg} / \mathrm{m}^{3}$ de fósforo $\mathrm{PO}_{4}-\mathrm{P}$

$C$ : Cantidad de fósforo al inicio $107680 \mathrm{~kg}$ de fósforo $\mathrm{PO}_{4}-\mathrm{P}$

Qe: caudal ingresante: $235 \mathrm{~kg} /$ día; equivalente a $0,25 \mathrm{~m}^{3} / \mathrm{día}$

$\mathrm{Ce}$ : concentración del contaminante en el flujo ingresante: $7,8867 \mathrm{~kg} / \mathrm{m}^{3}$

$K=$ tasa de sedimentación o tasa de remoción $0,36 /$ día

$V$ : volumen del cuerpo lentico: $3,295 \times 10^{9} \mathrm{~m}^{3}$ y H: profundidad: $25 \mathrm{~m}$

Reescribiendo la ecuación en la forma habitual para una ecuación diferencial lineal:

$$
\frac{\mathrm{dC}}{\mathrm{dt}}+\frac{\mathrm{kC}}{\mathrm{V}}=\mathrm{Qe} \mathrm{Ce}
$$

Por tanto, el factor de integración es $\mathrm{e}^{\mathrm{k} / \mathrm{V}} \mathrm{y}$ la solución general es:

$$
\mathrm{C}(\mathrm{t})=\underline{\mathrm{v}}+\mathrm{C} \mathrm{e}^{-\mathrm{kt} / \mathrm{V}}
$$


Donde $\mathrm{C}$ es una constante arbitraria, utilizando las condiciones iniciales $\mathrm{t}=0 \mathrm{y}$ $\mathrm{c}=107,680 \mathrm{~kg}$ de fósforo $\mathrm{PO}_{4}-\mathrm{P}$ y los valores señalados:

$$
\begin{aligned}
& 107680=\frac{3.295 \times 10^{9}}{0.36}+C \mathrm{e}^{-\left(0,36^{*}{ }^{*} / 3^{\prime 2} 295000000\right)} \\
& C=-9152^{\prime} 670098.03922 \mathrm{~kg}
\end{aligned}
$$

Entonces, la ecuación que predice la cantidad de P en kg en cualquier instante es:

$$
C(t)=9^{\prime} 152^{\prime} 777778-9152^{\prime} 670098,03922 \mathrm{e}^{-\left(0,36^{*} t^{\prime} / 3^{\prime 2} 295^{\prime} 000000\right)}
$$

Esta ecuación se utiliza para determinar la cantidad de fósforo en $\mathrm{kg}$ en función del tiempo. $\mathrm{Al}$ inicio de la investigación se calculó una cantidad de 107679 toneladas de fósforo en la laguna Arapa que tiene un volumen calculado de $3,295 \times 10^{9} \mathrm{~m}^{3}$, después de 365 días se registró una cantidad de 108044 toneladas, entonces el incremento de fósforo durante un año de producción de truchas fue de $365 \mathrm{~kg}$ que se han ido disolviendo en todo el volumen de agua de la laguna Arapa, Por otro lado, de la tabla 2 se tiene que la cantidad total de fósforo vertido al lago, producto de la digestión del alimento consumido, fue de $611 \mathrm{~kg}$. Con estos datos se estima que la diferencia que es de $246 \mathrm{~kg}$ de fósforo se destina a acumularse en los sedimentos.

La cantidad de fósforo que se ha incrementado en la laguna Arapa proviene exclusivamente de las heces de las truchas, luego del proceso de digestión del alimento. 


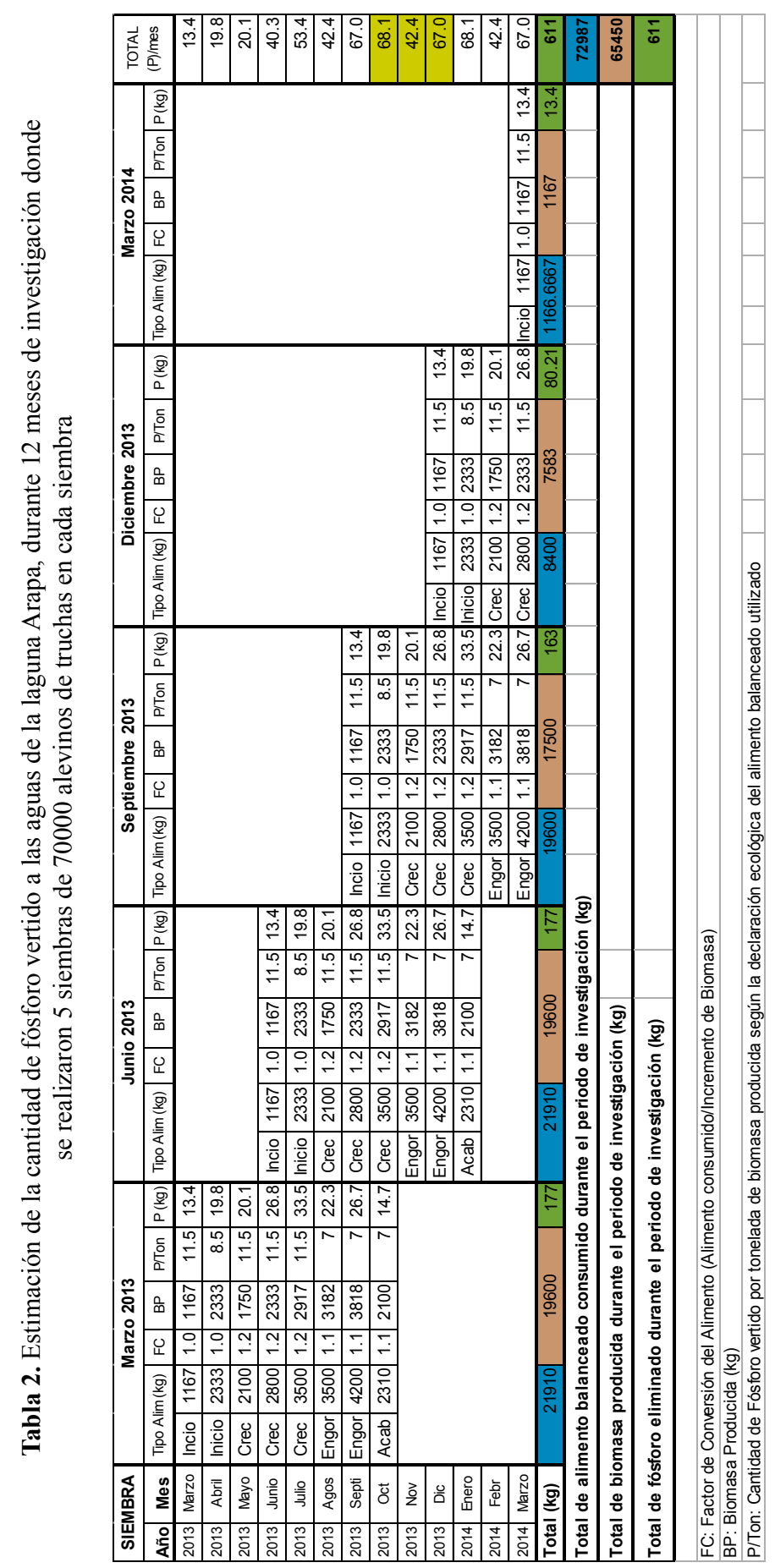




\section{CONCLUSIONES}

La evaluación de los parámetros indicadores de contaminación en la zona de producción de trucha en jaulas flotantes en la laguna Arapa, manifestaron diferencias significativas en los parámetros de acidez, dióxido de carbono, fosfatos y conductividad eléctrica; incrementándose los valores de estos parámetros. Por otro lado, los sólidos suspendidos totales demostraron una disminución. Con respecto a los parámetros de alcalinidad, $\mathrm{pH}$ y oxígeno disuelto, estos permanecieron constantes.

El principal insumo utilizado en la producción de trucha es el alimento balanceado y su incidencia en la contaminación corresponde a la cantidad total de fósforo vertido al lago, que fue de $611 \mathrm{~kg}$, producto de la digestión del alimento consumido durante el tiempo de investigación. Se utilizó $70478 \mathrm{~kg}$ de alimento balanceado distribuido en los diferentes tipos de presentación, y se alcanzó una biomasa de $65450 \mathrm{~kg}$ de trucha. La concentración de fósforo en la laguna Arapa se incrementó con la actividad de crianza de truchas, alcanzando valores de $32,79 \mathrm{mg} / \mathrm{m}^{3}$ de $\mathrm{PO}_{4}-\mathrm{P}$ que lo clasifican como lago eutrófico según la clasificación de Vollenweider.

Se determinó un modelo matemático simplificado para el fósforo, que predice la cantidad de fósforo en $\mathrm{kg}$ en cualquier instante. Después de 365 días de crianza de trucha se registró una cantidad de $611 \mathrm{~kg}$ de fósforo vertidos a la laguna Arapa, de los cuales $246 \mathrm{~kg}$ de fósforo se destinaron a acumularse en los sedimentos y $365 \mathrm{~kg}$ se fueron disolviendo en todo el volumen de agua de la laguna.

\section{AGRADECIMIENTO}

Los autores agradecen a la Empresa Arapa San Pedro y San Pablos S.A.C. por las facilidades para el desarrollo del presente trabajo de investigación en sus instalaciones.

\section{REFERENCIAS BIBLIOGRÁFICAS}

1. Buschmann A. Impacto ambiental de la acuicultura, el estado de la investigación en Chile y el mundo [Internet]. Osorno, Chile: Terram Publicaciones. 2015 [accesado 1 de setiembre de 2014]. Disponible en: http://www.cetmar.org/DOCUMENTACION/dyp/ ImpactoChileacuicultura.pdf

2. Soler M, Rodríguez H, Victoria P. Fundamentos de Nutrición y Alimentación en Acuicultura. Bogotá: INPA Instituto Nacional de Pesca y Acuicultura; 1996.

3. Ogino C, Yang G. Requirements of rainbow trout for dietary zinc. Bull Jap Soc Sci Fish. 1979; 44: 1015-18.

4. Restrepo C. Aproximación a la dinámica del transporte del nitrógeno y del fósforo en la ciénaga de Ayapel. [Tesis]. Medellin: Universidad Nacional de Colombia; 2005.

5. Steeter H, Phelps E. A study of the pollution and natural purification of the Ohio River, 
III [En línea]. Washington: Public Health Bulletin; 1958 [Consultado: 7 de Noviembre del 2014]. Disponible en: dspace.udel.edu/bitstream/handle/19716/1590/C\%26EE148. pdf? sequence $=2$

6. Thomann R, Mueller J. Principles of surface water quality modelling and control. New York: Harper \& row; 1987.

7. Masters G, Ela W. Introducción a la Ingeniería Medioambiental. Madrid: Pearson Educación S.A.; 2008.

8. López Y, Trujillo E, Fonseca G, Martínez V. Identificación de las características físicas, químicas y microbiológicas del agua, que se alteran por uso acuícola. México D.F.: Centro Interamericano de Recursos del Agua, Facultad de Ingeniería. Universidad Autónoma del Estado de México; 2005.

9. Blanco C. La trucha, cría industrial. 1a ed. Madrid: Ediciones Mundi-Prensa; 1995.

10. Orozco C, Pérez A, Gonzales M, Rodríguez F, Alfayate J. Contaminación ambiental. Una visión desde la química. Madrid: Thomson Editores Spain; 2003.

11. Cole G. Manual de Limnología. Buenos Aires: Editorial Hemisferio Sur; 1982.

12. Klontz G. Producción de trucha arcoíris en granjas familiares. México D.F.: Alimentos de Alta Calidad El Pedregal, S.A. de C.V.; 1991.

13. Putz P. Informe practico. Analítica de laboratorio y sistema de control de proceso. Eliminación y determinación de fosfatos. Vizcaya: HACH-LANGE; 2009.

14. Biomar [En línea]. Dinamarca: BioMar Group; c2014 [actualizada 15 de agosto 2014; consultado 15 de agosto 2014]. Disponible en: www.biomar.com

15. Breton B. El cultivo de la trucha. Barcelona: Ediciones Omega S.A.; 2007. 